\title{
Oral Infection - Parkinson's Disease, Concepts of Neuroinflammation. Could there be a New Investigation Model?
}

\author{
Ashank Mishra ${ }^{1 *}$, Jens Schwamborn ${ }^{2}$, Bart De Wever ${ }^{1}$ and Andrea Mascolo ${ }^{1}$ \\ ${ }^{1}$ Research Department, European Institute for Medical Studies (EIMS), Malta \\ ${ }^{2}$ Developmental and Cellular Biology, Luxembourg Centre for Systems Biomedicine (LCSB), University of \\ Luxembourg, Luxembourg
}

Periodontitis is a chronic, oral poly microbial infection affecting nearly $60 \%$ of the population worldwide and is the most prevalent oral inflammatory disease in adults. Periodontitis is not only an oral localized inflammatory disease, but also elicits low-grade systemic inflammation via both the release of pro-inflammatory cytokines and the invasion of periodontopathic bacteria. Besides inducing systemic inflammation, increasing evidence implies that periodontitis provokes chronic inflammation associated with activation of microglia, the immune cells in the brain, which is referred to as neuroinflammation. Neuroinflammation has become a common prominent feature among a variety of neurodegenerative disorders. Attention on the pathogenic role of neuroinflammation has, over the past two decades, been expanded to psychiatric disorders [1].

Based on the aforementioned findings, chronic inflammation can be regarded as a common denominator of periodontitis and neuropsychiatric disorders. Specifically, neuroinflammation may causally link periodontitis to the clinical onset and development of neuropsychiatric disorders. Furthermore, through the biological mechanism of chronic inflammation, periodontitis could causally affect neuropsychiatric disorders. The present review presents an overview of the evidence linking periodontitis with Parkinson's disease and calls for increased cooperation between dentists and medical doctors to provide optimal screening, treatment, and prevention of both Parkinson's disease and its comorbidities [2].

Oral infection/Periodontitis produces pro inflammatory mediators like IL-1, IL-6, TNF- $\alpha$. Due to persistent oral infection and saturation of cytokine receptors in oral mucosa, these mediators breach oral cavity and reach systemic circulation leading to systemic inflammatory storm. These systemic cytokines reach brain through multiple pathways i,e. Neural, humoral or cellular pathway. These mediators enter into brain through areas which lack Blood brain barrier (BBB) or crosses BBB causing changes in the permeability [3].

Systemic pro-inflammatory cytokines activate endothelial cells, expressing receptors for TNF- $\alpha$ and IL-1 $\beta$, which in turn, signal to the perivascular macrophages located immediately adjacent to endothelial cells. These perivascular macrophages subsequently communicate with microglia and thus lead to microglial activation. Activated microglia secretes not only pro-inflammatory cytokines but also proteases and chemokines, including monocyte chemo attractant protein (MCP)-1. MCP-1 is supposed to be responsible for the recruitment of monocytes into the motor cortex, hippocampus, and basal ganglia regions. Finally, all the inflammatory mediators and ROS species released by activated microglial cells increases the neurotoxicity and contributes to neurodegeneration of dopaminergic neurons of SN eventually causing Parkinson's disease.

Periodontal bacteria/bacterial molecules can directly invade the brain either through the blood stream or via cranial nerves. The leptomeninges could be a site of communication between periodontal bacteria and brain-resident microglia [4]. The leptomeninges covers the brain parenchyma surface and provides a physical boundary at the cerebrospinal fluid (CSF)-blood barrier. Leptomeningeal cells express Toll-like receptors (TLRs) 2 and 4 that are the receptors for $P$. gingivalis LPS. Leptomeningeal cells can be activated by circulating $P$. gingivalis LPS and subsequently produce pro-inflammatory cytokines for the brain. The pro-inflammatory cytokines released from leptomeningeal cells activate microglia to evoke neuroinflammation. Accordingly, the leptomeninges could be harmful by transducing peripheral inflammation, including periodontitis, into neuroinflammation.

*Corresponding author: Ashank Mishra, Research Department, European Institute for Medical Studies (EIMS), H.E.I., Malta

Accepted: February 13, 2021

Published online: February 15, 2021

Citation: Mishra A, Schwamborn J, Wever BD, et al. (2021) Oral Infection - Parkinson's Disease, Concepts of Neuroinflammation. Could there be a New Investigation Model?. J Oral Healthc 2(1):17-19

Copyright: (C) 2021 Mishra A, et al. This is an open-access article distributed under the terms of the Creative Commons Attribution License, which permits unrestricted use, distribution, and reproduction in any medium, provided the original author and source are credited. 


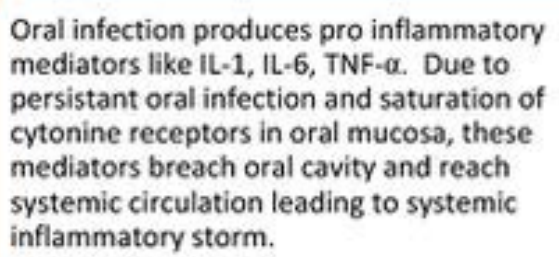

These pro inflammatory cytokines activate the perivasuclar macrophages which subsequently will communicate with microglial cells to activate them. activated microgial cells release MCP-1 (Monocute chemoattractant protein -1) which recruits monocytes/granulocytes in motor cortex, hippocampus and basal ganglion.

Finally, all the inflammatory mediators and ROS species released by activated microglial cells increases the neurotoxicity and contributes to neurogeneration of dopaminergic neurons of SN eventually causing Parkinsons Disease.

Figure 1: Oral infection-Parkinson's disease: Concepts of neuroinflammation.

We speculate that infection and inflammation caused by problems with oral health could be a trigger for PD. It might act like a second hit leading to disease onset in individuals who probably already have a genetic predisposition.

We further propose that human midbrain organoids could be an ideal test model to investigate this hypothesis. Human midbrain organoids can be generated from healthy individuals as well as from PD patients [5]. Importantly, for this model PD patient specific phenotypes, like reduced amounts of dopaminergic neurons as well appearance of alpha-Synuclein positive protein aggregates have been shown already $[6,7]$. Finally, a recent study showed that human midbrain organ- oids can be used to induce PD-like phenotypes by treatment with toxins [8]. A first line of experiments could be to generate midbrain organoids from various individuals (ideally patients and healthy controls) and treat them with the substances that are relevant in the context of oral healthy (e.g. (zinc and dental amalgam) as well as with pro-inflammatory mediators, in order to investigate their reaction. At various durations after treatment the amount of dopaminergic neurons as well as the aggregation of alpha-Synuclein could be analysed.

On the long run, by making use of organ-on-chips technology it might be very interesting to construct even more 
complex in vitro systems linking brain organoids and e.g. Dentin-Pulp-Like Organoids [9]. This would allow studying the potential interplay between oral health and PD in an unprecedented manner [10] (Figure 1).

\section{References}

1. Hashioka S, Inoue K, Miyaoka T, et al. (2019) The possible causal link of periodontitis to neuropsychiatric disorders: More than psychosocial mechanisms. Int J Mol Sci 20: 3723.

2. Holmstrup P, Damgaard C, Olsen I, et al. (2017) Comorbidity of periodontal disease: Two sides of the same coin? An introduction for the clinician. J Oral Microbiol 9: 1332710.

3. Ranjan R, Dhar G, Sahu S, et al. (2018) Periodontal disease and neurodegeneration: The possible pathway and contribution from periodontal infections. Journal of Clinical and Diagnostic Research 12: DE01-DE05.

4. Liu Y, Wu Z, Zhang X, et al. (2013) Leptomeningeal cells transduce peripheral macrophages inflammatory signal to microglia in response to Porphyromonas gingivalis LPS. Mediat Inflamm 2013: 407562.
5. Monzel AS, Smits LM, Hemmer K, et al. (2017) Derivation of human midbrain-specific organoids from neuroepithelial stem cells. Stem Cell Reports 8: 1144-1154.

6. Smits LM, Reinhardt L, Reinhardt P, et al. (2019) Modeling parkinson's disease in midbrain-like organoids. NPJ Parkinsons Dis 5: 5.

7. Kim H, Park HJ, Choi H, et al. (2019) Modeling G2019S-LRRK2 sporadic parkinson's disease in 3D midbrain organoids. Stem Cell Reports 12: 518-531.

8. Monzela AS, Hemmera K, Kaoma T, et al. (2020) Machine learning-assisted neurotoxicity prediction in human midbrain organoids. Parkinsonism Relat Disord 75: 105-109.

9. Jeong SY, Lee S, Choi WH, et al. (2020) Fabrication of dentinpulp-like organoids using dental-pulp stem cells. Cells 9: 642.

10. Kaur T, Uppoor A, Naik D (2016) Parkinson's disease and periodontitis - the missing link? A review. Gerodontology 33: 434438. 